\title{
Multilocus analysis of introgression between two sand fly vectors of leishmaniasis
}

\author{
Camila J Mazzoni ${ }^{1}{ }^{3}$, Alejandra S Araki ${ }^{1}$, Gabriel EM Ferreira1, \\ Renata VDM Azevedo ${ }^{1,4}$, Guido Barbujani ${ }^{2}$ and Alexandre A Peixoto*1
}

\begin{abstract}
Address: ${ }^{1}$ Laboratório de Biologia Molecular de Insetos, IOC, Fundação Oswaldo Cruz, Rio de Janeiro, Brazil, ${ }^{2}$ Dipartimento di Biologia, Università di Ferrara, Ferrara, Italy, ${ }^{3}$ Environmental Research Institute, University College Cork, Cork, Ireland and ${ }^{4}$ present address: Department of Genetics, University of Leicester, Leicester, UK

Email: Camila J Mazzoni - c.mazzoni@ucc.ie; Alejandra S Araki - saori@ioc.fiocruz.br; Gabriel EM Ferreira - gmelim@ioc.fiocruz.br; Renata VDM Azevedo - reazevedo@hotmail.com; Guido Barbujani - bjg@unife.it; Alexandre A Peixoto* - apeixoto@fiocruz.br

* Corresponding author
\end{abstract}

Published: 12 May 2008

BMC Evolutionary Biology 2008, 8:14| doi:|0.1|86/|47|-2|48-8-|4|

This article is available from: http://www.biomedcentral.com/|47|-2/48/8//4।

(c) 2008 Mazzoni et al; licensee BioMed Central Ltd.

This is an Open Access article distributed under the terms of the Creative Commons Attribution License (http://creativecommons.org/licenses/by/2.0), which permits unrestricted use, distribution, and reproduction in any medium, provided the original work is properly cited.
Received: 26 November 2007

Accepted: 12 May 2008

\begin{abstract}
Background: The phlebotomine sand flies (Diptera:Psychodidae) Lutzomyia (Nyssomyia) intermedia Lutz \& Neiva 1912 and Lutzomyia (Nyssomyia) whitmani Antunes \& Coutinho 1932 are two very closely related species and important vectors of American cutaneous leishmaniasis. Two singlelocus studies have revealed evidence for introgression between the two species in both mitochondrial and nuclear genomes. These findings have prompted the development of a multilocus approach to investigate in more detail the genetic exchanges between the two species.

Results: We analyzed ten nuclear loci using the "isolation with migration" model implemented in the IM program, finding evidence for introgression from $L$. intermedia towards $L$. whitmani in three loci. These results confirm that introgression is occurring between the two species and suggest variation in the effects of gene flow among the different regions of the genome.

Conclusion: The demonstration that these two vectors are not fully reproductively isolated might have important epidemiological consequences as these species could be exchanging genes controlling aspects of their vectorial capacity.
\end{abstract}

\section{Background}

Gene flow between closely related species has been reported in an increasing number of studies as a rule and not as an exception and it is currently well accepted that sibling species can retain a low level of gene flow between them [1]. In this case, divergence between closely related species is determined by competition between gene flow and genetic drift, where the first tends to decrease divergence, and the latter to increase it [2]. A number of studies have provided evidence that introgression can occur more easily in certain regions of the genome. This is determined mainly by natural selection, which is expected to restrain gene flow at regions associated with species-specific adaptations [3].

In insect disease vectors, gene flow between species may have important epidemiological consequences, as it might allow changes in the disease patterns. Fonseca et al. [4] have shown that hybrids between two different forms in the Culex pipiens complex, probably introduced in the United States at two different moments, may act as a bridge vector between birds and humans of the West Nile virus, contributing to the current epidemics. In another 
example, Besansky et al. [5] have proposed that the acquisition of chromosome inversions by Anopheles gambiae from the more arid-adapted Anopheles arabiensis may have contributed to the spread and ecological dominance of this malaria vector.

Evidence of introgression has also been reported in sand flies [6,7], including Lutzomyia intermedia and Lutzomyia whitmani two important vectors of cutaneous leishmaniasis in Brazil [8]. Recently, we obtained further evidence for gene flow between these two closely related species using the period (per) gene [9], a locus controlling circadian and lovesong rhythms in Drosophila, that might have a role in the reproductive isolation between sibling species [reviewed in [10]]. These first pieces of evidence for introgression lead us to inquire about the level and extent of gene flow between $L$. intermedia and $L$. whitmani.

In this study, we performed a multilocus analysis using ten different nuclear loci in a sample of $L$. intermedia and L. whitmani from the locality of Afonso Claudio (Southeast Brazil) in order to estimate the level of gene flow between these two vector species in each individual locus and across the genome. The possibility that introgression between these two leishmaniasis vectors is extensive and recurrent, could have important epidemiological consequences. For example, genes controlling aspects of vectorial capacity such as adaptation to man made habitats (domestic and peridomestic areas), competence to transmit different Leishmania strains and host preference could be passing from one species to another [8]. Analysis of multiple markers might determine if introgression between the two species is affecting many loci and whether gene flow in genes known to control aspects of the reproductive isolation in other species, such as period, is relatively reduced compared to other loci.

\section{Results}

\section{Molecular markers}

The choice of molecular markers includes genes with different functions and genome locations as described in the Methods. The ten loci used in this study are the homologues of the following Drosophila genes: $\mathrm{Ca1D}$ (or $\mathrm{Ca}$ $\alpha 1 D$; $\mathrm{Ca}^{2+}$-channel protein $\alpha_{1}$ subunit D), cac (cacophony), Rp49 (or RpL32; Ribosomal protein L32), RpL17A (or RpL23; Ribosomal protein L23), RpL36 (Ribosomal protein L36), RpS19a (Ribosomal protein S19a), TfIIA-L (Transcription factor IIA L), up (upheld) and $\zeta$ COP (or zetacop) [11]. In addition, we also obtained new sequences of per from the same samples. Sequences have been submitted to Genbank (accession numbers EU082834-EU083311).

\section{Polymorphism, Recombination and Divergence Analyses}

Initially all sequences were checked for recombination, a necessary step for the IM analysis (see below). The four- gamete test [12] was carried out for each locus in order to identify fragments with no evidence for internal recombination events. The larger non-recombining (NR) block including at least part of an intron was finally chosen for each locus to be used in subsequent population analyses (see Methods).

Table 1 shows the minimum number of recombination events for each gene and summarizes polymorphism information for both the NR block and the whole fragment at each locus. It is noticeable that some of the loci have a large difference in length and in the number of segregating sites between the whole fragment and the NR block, but this is often due to the high number of recombination events identified in some loci. However, in general, this difference does not affect the level of per nucleotide polymorphism, except for an increase of $\pi$ and $\theta$ in the per and cac NR blocks, which is probably due to differences in evolutionary rates between different parts of these gene fragments.

Divergence between $L$. intermedia and $L$. whitmani, estimated from $F_{s t}$ values, is relatively high (Table 2), ranging from 0.17 to 0.64 , except for zetacop, which shows limited divergence between the two species $\left(F_{s t}=0.0542\right.$ for the NR block, non significant). However, only three out of the ten loci present fixed differences (Rp49, RpL36 and $R p S 19 a)$, whereas polymorphisms are shared in most genes, particularly in per (14 shared polymorphisms for the whole fragment). Some of the loci present differences in the degree of genetic divergence estimated from the analysis of the whole fragment and the NR block, respectively. The RpL17A NR block presents far less divergence between species compared to the whole fragment $\left(F_{s t}=\right.$ 0.1749 and 0.4135 , respectively), while the opposite is observed in RpL36 ( $F_{s t}=0.6026$ and 0.3609 for the NR block and the whole fragment, respectively).

\section{IM Analysis}

An analysis of population divergence under the "Isolation with Migration" model has been performed using the IM software [2]. The IM analysis requires sequence data from individual loci that show variation within or between two populations, under the assumption that recombination is negligible. For this reason, only the NR blocks containing at least part of an intron have been used for the analyses and some putatively recombinant sequences were excluded (see Methods). In order to avoid mistaking the effects of selection for those of drift or migration, we carried out preliminary tests of selective neutrality. These included Tajima's D [13], Fu and Li's D* and F* [14], Ramos-Onsins and Rozas' $R_{2}$ [15], and Fu's $F_{S}$ [16]. The statistic tests have been calculated using the DnaSP software [17] (for Tajima's D estimates see Table 1; for the other tests see additional file 1). The simulation analyses 
Table I: Polymorphism analyses for each locus/non-recombining block

\begin{tabular}{|c|c|c|c|c|c|c|c|c|}
\hline locus & species & $\mathrm{n}^{\mathrm{a}}$ & Length(bp) & $\mathrm{RM}^{\mathrm{b}}$ & Sc & $\pi^{\mathrm{d}}$ & $\theta^{\mathrm{e}}$ & Tajima's Df \\
\hline \multirow[t]{2}{*}{ CaID } & L.intermedia & $27(25)$ & $176(176)$ & 1 & $6(5)$ & $0.0|4|(0.0129)$ & $0.0088(0.0075)$ & I.725I (2.0390) \\
\hline & L.whitmani & $28(24)$ & & & $13(11)$ & $0.0205(0.0185)$ & $0.0190(0.0167)$ & $0.2687(0.354 I)$ \\
\hline \multirow[t]{2}{*}{$\mathrm{cac}$} & L.intermedia & $12(11)$ & $\mid 72(5 \mid)$ & 2 & $4(3)$ & $0.0100(0.0235)$ & $0.0077(0.0201)$ & $1.029 \mid(0.5873)$ \\
\hline & L.whitmani & $30(28)$ & & & $9(8)$ & $0.0104(0.0306)$ & $0.0132(0.0403)$ & $-0.6517(-0.7434)$ \\
\hline \multirow[t]{2}{*}{ per } & L.intermedia & $21(20)$ & $481(86)$ & 13 & $29(7)$ & $0.0154(0.0204)$ & $0.0168(0.0229)$ & $-0.4254(-0.3674)$ \\
\hline & L.whitmani & $23(22)$ & & & $36(12)$ & $0.0236(0.03 \mid 2)$ & $0.0203(0.0383)$ & $0.2943(-0.8805)$ \\
\hline \multirow[t]{2}{*}{$R p 49$} & L.intermedia & $24(18)$ & $237(237)$ & 5 & $13(12)$ & $0.0149(0.0135)$ & $0.0147(0.0147)$ & $0.0440(-0.3030)$ \\
\hline & L.whitmani & $17(16)$ & & & $5(5)$ & $0.0040(0.0035)$ & $0.0062(0.0064)$ & $-1.14 \mid 2(-1.4912)$ \\
\hline \multirow[t]{2}{*}{ RpLI TA } & L.intermedia & $20(16)$ & $238(114)$ & 5 & $13(5)$ & $0.0188(0.0135)$ & $0.0154(0.0132)$ & $0.4893(0.0580)$ \\
\hline & L.whitmani & $3 I(3 \mid)$ & & & $19(8)$ & $0.0162(0.0101)$ & $0.0200(0.0176)$ & $-0.9231(-1.2859)$ \\
\hline \multirow[t]{2}{*}{$R p L 36$} & L.intermedia & $36(34)$ & $4 \mid 2(|| 3)$ & 13 & $29(9)$ & $0.0164(0.0185)$ & $0.0170(0.0195)$ & $-0.1108(-0.1489)$ \\
\hline & L.whitmani & $11(I I)$ & & & $29(6)$ & $0.0279(0.0183)$ & $0.0240(0.0181)$ & $0.3898(0.0468)$ \\
\hline \multirow[t]{2}{*}{ RpSI9a } & L.intermedia & $36(34)$ & $194(117)$ & 3 & $9(5)$ & $0.0091(0.0106)$ & $0.0112(0.0105)$ & $-0.5708(0.0462)$ \\
\hline & L.whitmani & $48(46)$ & & & $14(5)$ & $0.0169(0.0119)$ & $0.0163(0.0097)$ & $-0.0895(0.0532)$ \\
\hline \multirow[t]{2}{*}{ TfIIA-L } & L.intermedia & $23(23)$ & $355(254)$ & 1 & $13(10)$ & $0.008 I(0.0080)$ & $0.0099(0.0107)$ & $-0.6493(-0.8337)$ \\
\hline & L.whitmani & $2 I(2 I)$ & & & $11(I I)$ & $0.0115(0.0161)$ & $0.0086(0.0120)$ & I.1776(I.1776) \\
\hline \multirow[t]{2}{*}{ up } & L.intermedia & $11(11)$ & $428(354)$ & 3 & $6(2)$ & $0.004 I(0.0010)$ & $0.0048(0.0019)$ & $-1.0943(-1.4296)$ \\
\hline & L.whitmani & $I I(I I)$ & & & $22(14)$ & $0.0172(0.0127)$ & $0.0176(0.0135)$ & $-0.4745(-0.778 I)$ \\
\hline \multirow[t]{2}{*}{ zetacop } & L.intermedia & $29(28)$ & $292(230)$ & 3 & $10(8)$ & $0.0056(0.0059)$ & $0.0087(0.0089)$ & $-1.1477(-1.0613)$ \\
\hline & L.whitmani & $19(18)$ & & & $17(14)$ & $0.0110(0.0116)$ & $0.0167(0.0177)$ & $-1.2924(-1.2976)$ \\
\hline
\end{tabular}

Note: values refer to sequences without gaps/ambiguous alignment; numbers in parentheses are related to the non-recombining block for each locus.

aNumber of sequences for each species for each locus.

bThe minimum number of recombination events.

cNumber of segregating sites.

dThe average number of nucleotide differences per site between two sequences.

eTheta $(\theta)=4 \mathrm{~N} \mu$ from $S$ (number of segregating sites).

fNone of the Tajima's D values was significant after Bonferroni's correction.

of the HKA software [18] were used for performing the HKA multilocus test of neutral molecular evolution [19]. In only one case (NR block of zetacop in L. whitmani for the $\mathrm{F}_{\mathrm{S}}$ test) a significant deviation from neutrality has been detected after Bonferroni's correction (see additional file
1). Based on their chromosome positions in Drosophila [11], all ten loci studied are expected to be unlinked, although we do not know their location in the Lutzomyia genome.

Table 2: Divergence between $L$. intermedia and $L$. whitmani at each locus, whole fragment and non-recombining block

\begin{tabular}{|c|c|c|c|c|c|c|}
\hline locus & $F_{s t}^{a}$ & $\mathrm{Nm}^{\mathrm{b}}$ & Dxyc & $\mathrm{Da}^{\mathrm{d}}$ & Fixede & Shared \\
\hline CaID & $0.4139 *\left(0.4567^{*}\right)$ & $0.354(0.2974)$ & $0.0295(0.0289)$ & $0.0122(0.0132)$ & $0(0)$ & $2(I)$ \\
\hline$c a c$ & $0.2874 *\left(0.2593^{*}\right)$ & $0.6198(0.7142)$ & $0.0143(0.0365)$ & $0.004 I(0.0095)$ & $0(0)$ & $1(I)$ \\
\hline per & $0.3188^{*}\left(0.3313^{*}\right)$ & $0.534 I(0.5045)$ & $0.0288(0.0386)$ & $0.0092(0.0128)$ & $0(0)$ & $14(1)$ \\
\hline$R p 49$ & $0.6144^{*}\left(0.6407^{*}\right)$ & $0.1569(0.1402)$ & $0.0244(0.0236)$ & $0.015(0.0151)$ & $\mathrm{I}(\mathrm{I})$ & $I(0)$ \\
\hline RpLI TA & $0.4135 *(0.1749 *)$ & $0.3546(1.1798)$ & $0.0301(0.014)$ & $0.0126(0.0022)$ & $0(0)$ & $7(1)$ \\
\hline RpL36 & $0.3609 *(0.6026 *)$ & $0.4427(0.1649)$ & $0.036(0.0464)$ & $0.0138(0.028)$ & $2(2)$ & $6(0)$ \\
\hline RpSI9a & $0.4593 *\left(0.56 \mid 8^{*}\right)$ & $0.2943(0.195)$ & $0.024(0.0257)$ & $0.011(0.0145)$ & $\mathrm{I}(\mathrm{I})$ & $3(I)$ \\
\hline TfIIA-L & $0.4401 *(0.3646 *)$ & $0.3181(0.4357)$ & $0.0174(0.0188)$ & $0.0076(0.0067)$ & $0(0)$ & $0(0)$ \\
\hline up & $0.3562 *(0.4168 *)$ & $0.4518(0.3499)$ & $0.0167(0.012)$ & $0.006 I(0.005 I)$ & $0(0)$ & $3(I)$ \\
\hline zetacop & $0.0552^{+}\left(0.0542^{\text {ns }}\right)$ & $4.2752(4.362 I)$ & $0.0088(0.0092)$ & $0.0005(0.0005)$ & $0(0)$ & $3(1)$ \\
\hline
\end{tabular}

aPairwise fixation index. Significance evaluated with 10000 permutations; * significant at $P<0.00$ I;

+ significant at $P<0.01$; ns non significant $P>0.01$.

$\mathrm{b}$ Estimated number of migrants per generation between populations calculated from $F_{s t}$.

cThe average number of nucleotide substitutions per site between populations.

dThe number of net nucleotide substitutions per site between populations.

e Number of fixed differences between species.

fNumber of shared polymorphic sites between species. 
Table 3: Model parameter estimates for all loci

\begin{tabular}{|c|c|c|c|c|c|c|c|c|c|c|c|c|c|c|c|c|c|c|}
\hline & \multicolumn{3}{|c|}{$\theta_{1}{ }^{\mathrm{a}}$} & \multicolumn{3}{|c|}{$\theta_{2}^{\mathrm{a}}$} & \multicolumn{3}{|c|}{$\theta_{\mathrm{A}}^{\mathrm{a}}$} & \multicolumn{3}{|c|}{$t^{\mathrm{b}}$} & \multicolumn{3}{|c|}{$m_{1}{ }^{c}$} & \multicolumn{3}{|c|}{$m_{2}{ }^{c}$} \\
\hline & $\mathbf{a}$ & b & c & $\mathbf{a}$ & b & c & $\mathbf{a}$ & b & c & $\mathbf{a}$ & b & c & $\mathbf{a}$ & b & c & $\mathbf{a}$ & b & c \\
\hline Minbind & 1.236 & 1.263 & 1.453 & 0.714 & 0.790 & 0.790 & 0.014 & 0.014 & 0.014 & 0.578 & 0.593 & 0.588 & 0.002 & 0.002 & 0.002 & 0.002 & 0.002 & 0.002 \\
\hline Maxbind & 7.320 & 6.939 & 7.374 & 4.024 & 4.043 & 3.986 & 5.283 & 7.238 & 6.043 & 2.733 & 2.783 & 3.038 & 0.814 & 0.734 & 0.810 & 0.678 & 0.650 & 0.630 \\
\hline $\mathrm{HiPt}^{\mathrm{e}}$ & 2.838 & 2.811 & 2.920 & 1.608 & 1.684 & 1.589 & 0.448 & 0.475 & 0.557 & 1.348 & 1.293 & 1.328 & 0.102 & 0.094 & 0.094 & 0.002 & 0.002 & 0.002 \\
\hline HiSmthe & 2.838 & 2.838 & 2.838 & 1.646 & 1.665 & 1.646 & 0.475 & 0.503 & 0.530 & 1.328 & 1.278 & 1.313 & 0.098 & 0.094 & 0.094 & 0.002 & 0.002 & 0.002 \\
\hline Mean & 2.947 & 2.947 & 2.947 & 1.703 & 1.703 & 1.684 & 0.638 & 0.665 & 0.638 & 1.338 & 1.323 & 1.323 & 0.118 & 0.114 & 0.114 & 0.030 & 0.030 & 0.030 \\
\hline 95Lof & 2.078 & 2.078 & 2.105 & 1.189 & 1.189 & 1.189 & 0.041 & 0.068 & 0.041 & 0.918 & 0.913 & 0.908 & 0.018 & 0.022 & 0.018 & 0.002 & 0.002 & 0.002 \\
\hline $95 \mathrm{Hi}^{f}$ & 4.115 & 4.142 & 4.142 & 2.407 & 2.407 & 2.407 & 1.833 & 1.833 & 1.860 & 1.848 & 1.828 & 1.843 & 0.302 & 0.298 & 0.294 & 0.174 & 0.170 & 0.170 \\
\hline HPD90Log & 2.051 & 2.051 & 2.078 & 1.151 & 1.151 & 1.151 & 0.014 & 0.041 & 0.014 & 0.958 & 0.943 & 0.938 & 0.014 & 0.014 & 0.014 & 0.002 & 0.002 & 0.002 \\
\hline HPD90Hig & 3.870 & 3.897 & 3.925 & 2.274 & 2.274 & 2.274 & 1.399 & 1.426 & 1.426 & 1.743 & 1.718 & 1.723 & 0.242 & 0.238 & 0.238 & 0.110 & 0.106 & 0.110 \\
\hline
\end{tabular}

Note: Values are presented for each of the three runs with different seed numbers ( $a, b$ and $c)$.

a The population size parameter for $L$. whitmani $\left(\theta_{1}\right)$, L. intermedia $\left(\theta_{2}\right)$ and ancestral population $\left(\theta_{\mathrm{A}}\right) ; \theta=4 \mathrm{Nm}$.

b Time of population splitting parameter

c Migration rate estimate ( $m_{1}-$ from $L$. intermedia to $L$. whitmani; $m_{2}-$ from $L$. whitmani to $L$. intermedia).

dThe midpoint value of the lowest (Minbin) and highest (Maxbin) bin.

e The value of the bin with the highest count ( $\mathrm{HiPt})$, after the counts have been smoothed by taking a running average of 9 points centered on each bin (HiSmth).

f The estimated points to which $2.5 \%$ of the total area lies to the left $(95 \mathrm{Lo})$ and to the right $(95 \mathrm{Hi})$.

$\mathrm{g}$ The lower (HPD90Lo) and upper (HPD90Hi) bound of the estimated $90 \%$ highest posterior density (HPD) interval.

Migration parameters have been estimated for each locus as well as for all loci together for each population in different IM runs. Our aim was to detect the occurrence of gene flow using the multilocus data, and determine whether the evidence for introgression is exclusive to some loci. All marginal densities suggest stationary distributions, with one exception (see below).

The marginal posterior probability densities for each of the six demographic parameters estimated using IM are shown in Figure 1. The results obtained suggest gene flow from L. intermedia to L. whitmani (Figure 1, top right graph). Table 3 summarizes the features from the mar- ginal histograms for each of the parameters. The migration rate estimate with the highest smoothed value of likelihood is $m_{1}=0.095$ (average of 3 independent runs) and the 95\% confidence intervals exclude the value zero for gene flow (Table 3). On the other hand, no evidence of migration has been found in the other direction, from L. whitmani to L. intermedia $\left(m_{2}=0.002\right.$, that is $m_{2} \sim 0$ as 0.002 is the lowest value that can possibly be estimated by the program under the conditions used).

Simulations were also carried out to estimate the migration rates of each locus separately. Figure 2 shows the marginal posterior probability densities for each locus
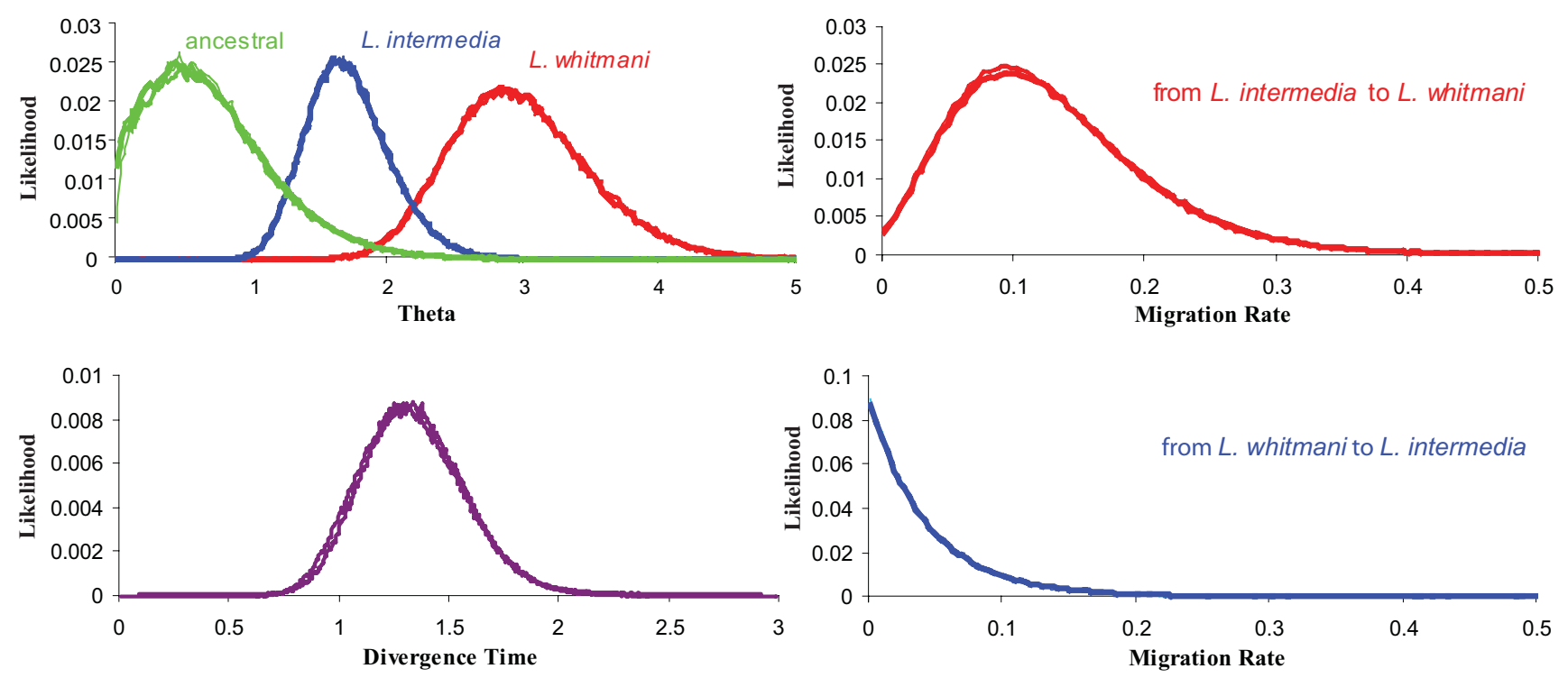

Figure I

Estimates of demographic parameters. Marginal Posterior Probability Densities for each of the six demographic parameters estimated using IM: theta $(\theta=4 \mathrm{~N} \mu)$; migration rate $(m=\mathrm{m} / \mu)$ and divergence time between species $(t=t \mu)$. Three IM simulations ( $a, b$ and $c$ ) with different seed numbers have been plotted for each parameter estimate (see also Table 3 ). 
Table 4: Migration parameter estimates for each locus

\begin{tabular}{|c|c|c|c|c|c|c|c|c|c|c|c|c|c|c|c|c|c|c|c|c|}
\hline & \multicolumn{2}{|c|}{$\underline{\text { CaID }}$} & \multicolumn{2}{|c|}{$\underline{c a c}$} & \multicolumn{2}{|c|}{ per } & \multicolumn{2}{|c|}{$\underline{R p 49}$} & \multicolumn{2}{|c|}{ RpLI7A } & \multicolumn{2}{|c|}{ RpL36 } & \multicolumn{2}{|c|}{ RpSI9a } & \multicolumn{2}{|c|}{ TfllA-L } & \multicolumn{2}{|c|}{$\underline{u p}$} & \multicolumn{2}{|c|}{ zetacop } \\
\hline & 1 & W & 1 & $w$ & $I$ & $w$ & 1 & $w$ & 1 & $w$ & $I$ & W & 1 & $w$ & 1 & $w$ & 1 & $w$ & $I$ & $w$ \\
\hline Minbin $^{\mathrm{a}}$ & 0.01 & 0.01 & 0.01 & 0.01 & 0.01 & 0.01 & 0.01 & 0.01 & 0.01 & 0.01 & 0.01 & 0.01 & 0.01 & 0.01 & 0.01 & 0.01 & 0.01 & 0.01 & 0.01 & 0.01 \\
\hline Maxbina & 13.54 & 12.21 & 19.99 & 19.99 & 15.93 & 12.93 & 19.99 & 18.91 & 19.99 & 19.99 & 16.45 & 15.88 & 5.45 & 4.04 & 15.37 & 9.87 & 19.99 & 19.99 & 19.99 & 19.99 \\
\hline $\mathrm{HiPt}^{\mathrm{b}}$ & 0.07 & 0.01 & 0.01 & 0.64 & 0.04 & 0.01 & 0.01 & 0.01 & 0.03 & 4.26 & 0.01 & 0.01 & 0.01 & 0.01 & 0.01 & 0.01 & 0.01 & 0.01 & 0.01 & 2.41 \\
\hline HiSmthb & 0.05 & 0.01 & 0.01 & 0.63 & 0.02 & 0.01 & 0.01 & 0.01 & 0.01 & 3.95 & 0.01 & 0.01 & 0.01 & 0.01 & 0.01 & 0.01 & 0.01 & 0.01 & 0.01 & 2.48 \\
\hline Mean & 0.41 & 0.23 & 0.91 & 1.25 & 0.46 & 0.32 & 0.31 & 0.25 & 6.18 & 8.00 & 0.21 & 0.23 & 0.17 & 0.11 & 0.25 & 0.24 & 0.44 & 0.41 & 0.83 & 3.63 \\
\hline 95Loc & 0.01 & 0.01 & 0.03 & 0.11 & 0.01 & 0.01 & 0.01 & 0.01 & 0.15 & 0.56 & 0.01 & 0.01 & 0.01 & 0.01 & 0.01 & 0.01 & 0.01 & 0.01 & 0.03 & 0.36 \\
\hline $95 \mathrm{Hic}^{\mathrm{c}}$ & 1.98 & 1.38 & 9.33 & 7.36 & 2.35 & 1.97 & 4.99 & 1.90 & 19.16 & 19.17 & 1.44 & 1.70 & 1.01 & 0.69 & 1.64 & 1.42 & 5.45 & 5.13 & 9.07 & 14.35 \\
\hline HPD90Lod & 0.01 & 0.01 & 0.01 & 0.01 & 0.01 & 0.01 & 0.01 & 0.01 & 0.01 & 0.01 & 0.01 & 0.01 & 0.01 & 0.01 & 0.01 & 0.01 & 0.01 & 0.01 & 0.01 & 0.01 \\
\hline HPD90 $\mathrm{Hi}^{d}$ & 1.24 & 0.80 & 4.15 & 3.76 & 1.43 & 1.10 & 1.67 & 0.94 & $18.6 ?$ & $16.9 ?$ & 0.76 & 0.88 & 0.61 & 0.43 & 0.90 & 0.82 & 1.94 & 1.78 & 4.07 & 9.13 \\
\hline
\end{tabular}

Note. I - L. intermedia; W - L. whitmani. Each estimated value is an average of four IM runs with different seed numbers.

a The midpoint value of the lowest (Minbin) and highest (Maxbin) bin.

${ }^{b}$ The value of the bin with the highest count (HiPt), after the counts have been smoothed by taking a running average of 9 points centered on each bin (HiSmth).

c The estimated points to which $2.5 \%$ of the total area lies to the left $(95 \mathrm{Lo})$ and to the right $(95 \mathrm{Hi})$.

d The lower (HPD90Lo) and upper (HPD90Hi) bound of the estimated $90 \%$ highest posterior density (HPD) interval.

* HPD estimate not reliable

migration rate while Table 4 presents a summary from the marginal histograms. The results have revealed nonzero values in three different loci, cac, RpL17A and zetacop towards L. whitmani (Figure 2 and Table 4), showing no evidence of introgression between these two species at the other loci. Although the distribution for the RpL17A locus does not reach the zero value on its right tail, this gene shows the highest estimate for the migration parameter in the direction of L. whitmani.

Maximum likelihood estimates for $\theta$ suggest that the $L$. whitmani effective population size is almost twice as large as in L. intermedia (Figure 1) while the ancestral population is estimated to be smaller than the current ones, indicating a possible expansion for both species.

Divergence time as well as the other parameters estimated with IM cannot be directly converted to numbers in years or generations, since the mutation rates in these two species or in other sand flies are unknown. However, using $D$. melanogaster synonymous and nonsynonymous substitution rates for nuclear genes, $1.56 \times 10^{-8}$ and $1.91 \times 10^{-9}$ per site per year [20], respectively, seems to be a reasonable first step for estimating the divergence time between Lutzomyia species. Thus, our educated guess for the divergence time between L. intermedia and L. whitmani would be of approximately 800 thousand years.

\section{Genealogy Analysis}

Gene trees for both entire sequence and NR block were estimated using Neighbor-Joining (NJ) and Parsimony methods, available in MEGA 3.1 [21], with similar results (data not shown). Only in the RpL36 gene tree the sequences of the two species occurred in two separate clusters (see below) while for 3 genes only one species formed a cluster (RpS19a, TfIIa-L and Rp49) and 5 genes did not present the sequences from any of the species in a single cluster (Ca1D, cac, per, up and zetacop) (data not shown).

Figure 3 presents the NJ trees from the NR blocks of the three loci that presented a nonzero migration estimate in the IM simulations: cac, RpL17A and zetacop. These trees are compared to the NJ tree of the RpL36 NR block (bottom right). While the former trees present non-structured topologies with a few identical haplotypes between the two species, the RpL36 NR block tree clearly groups both species in different clusters with a high bootstrap value $(86 \%)$. Very similar results were obtained when haplotype networks were estimated using BioNumerics v. 5.0 (Applied Maths). Figure 4 shows Minimum Spanning Trees of RpL36, cac, RpL17A and zetacop NR blocks. Again only RpL36 presents a clear separation between the haplotypes of the two species.

\section{Discussion}

Populations seldom evolve according to a simple and easy-to-identify mechanism. Under the model of Isolation with Migration, information on both divergence time and gene flow rates can be extracted from the data; thus, this model is more flexible and realistic than most alternative models of subdivided populations, which assume either absence of gene flow or an infinite divergence time $[2,22]$. Therefore, the IM model is particularly suitable for the study of recently-separated populations that may still be connected by some genetic exchanges, as we believe to be the case for the closely related species $L$. intermedia and L. whitmani, which despite presenting some identical haplotypes and no fixed differences in the per gene and in a mitochondrial marker $[8,9]$ are nevertheless distinguishable by morphological differences [23]. Indeed, the simulations presented here between these two vectors showed good convergence and consistency among independent runs. In addition, the estimated population size difference 


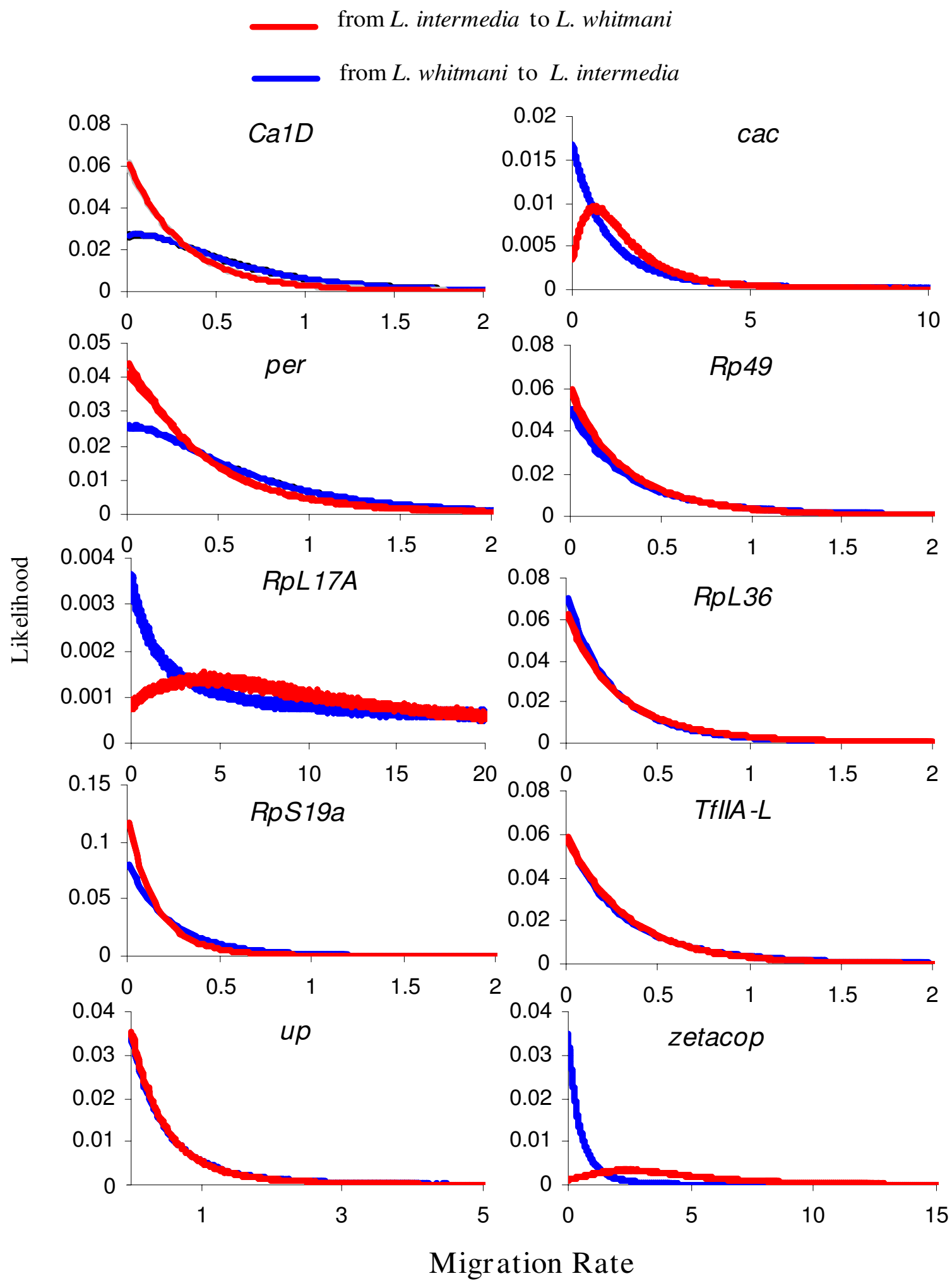

Figure 2

Migration rate estimates in 10 different loci. Marginal Posterior Probability Densities for each locus migration rate. Four IM simulations with different seed numbers have been plotted. 

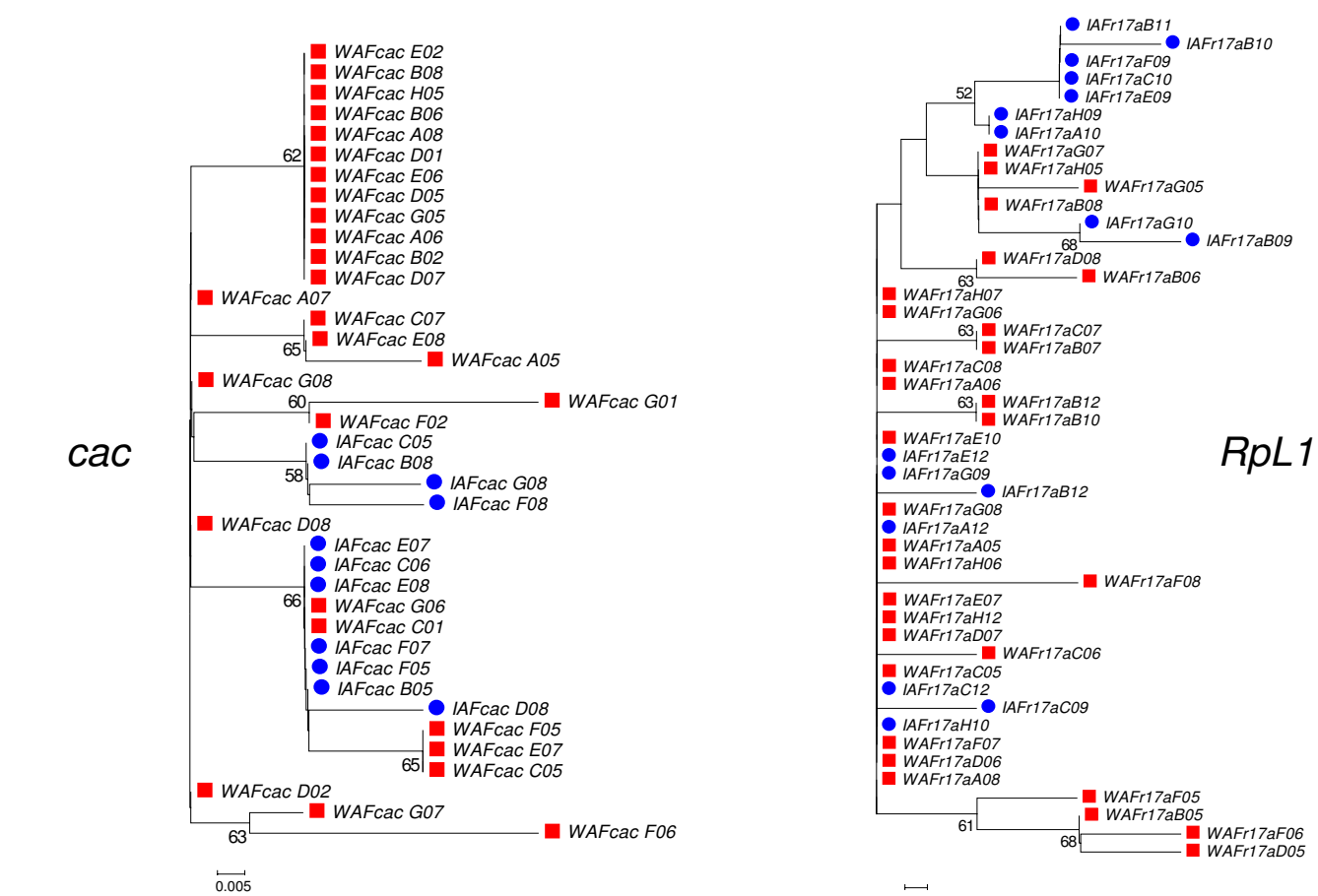

$\overleftarrow{0.00}$

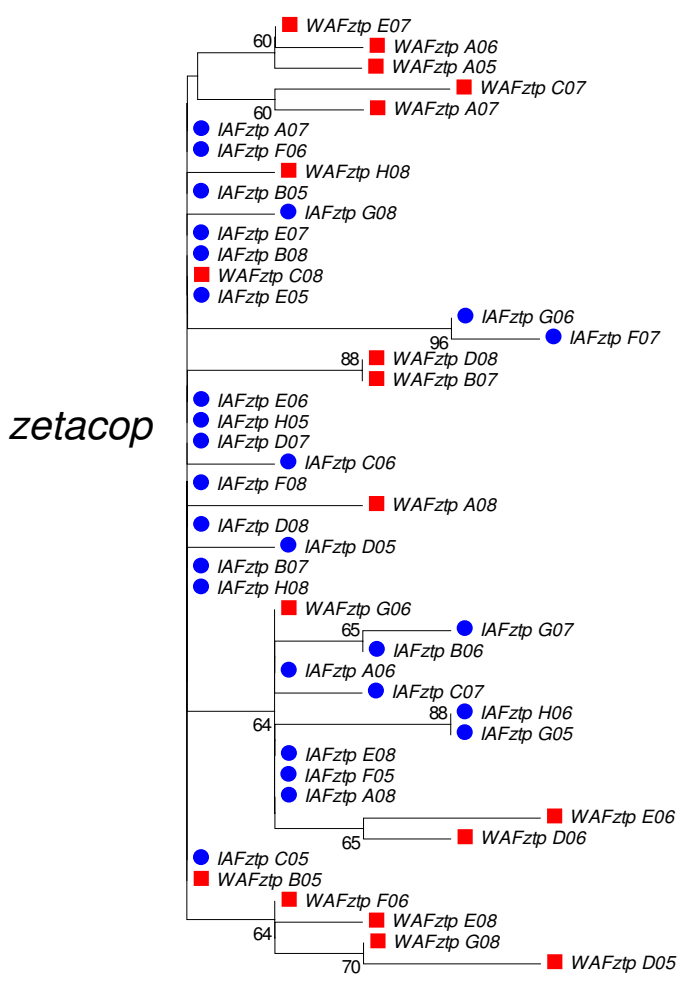

$\stackrel{\leftrightarrow 002}{0.02}$

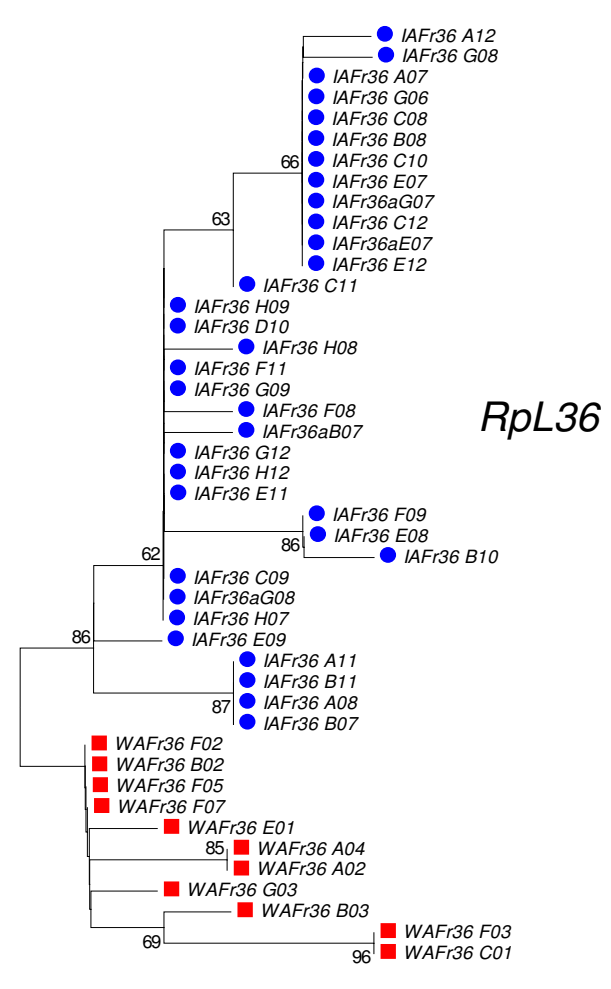

$\breve{0.005}$

Figure 3

Genealogies of NR blocks of four selected loci. Genealogies from the NR blocks of the three loci with evidence of gene flow (cac, RpLI 7A and zetacop) and one locus (RpL36) presenting fixed differences between $L$. intermedia (blue circles) and $L$. whitmani (red squares). The trees were estimated using the neighbor-joining method, Kimura-2-parameters distance and 1000 bootstrap replicates. The trees were rooted using the middle-point between the two most distant sequences. 

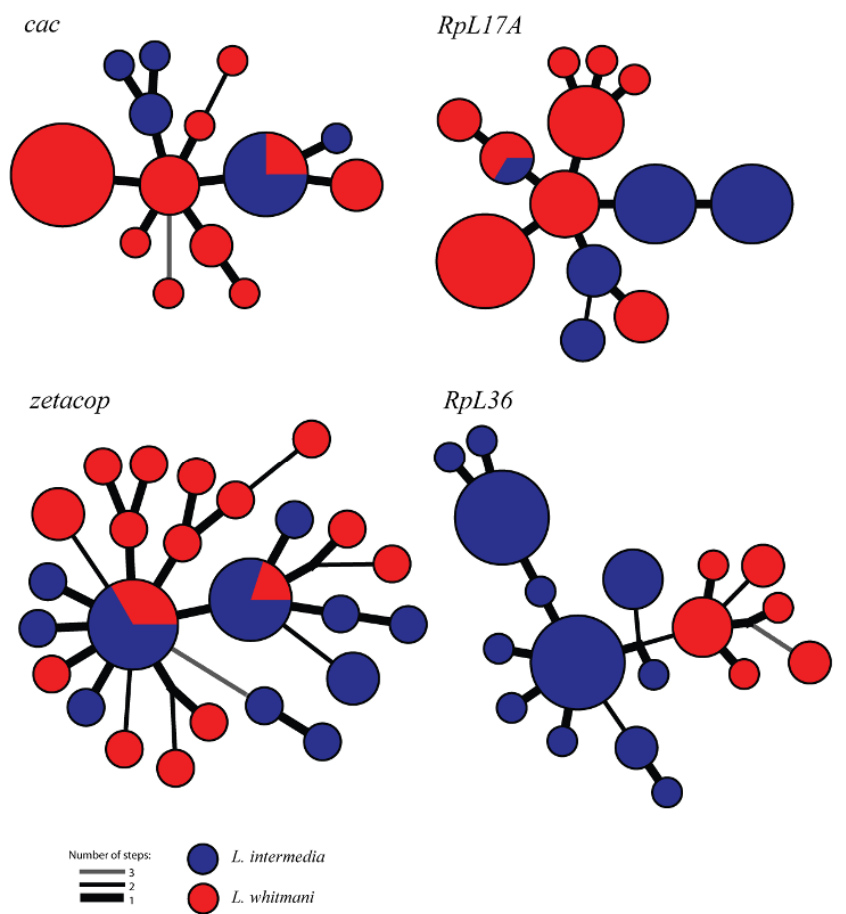

Figure 4

Haplotype Networks of NR blocks of four selected loci. Minimum spanning trees of the NR blocks of the three loci with evidence of gene flow (cac, RpLI 7A and zetacop) and one locus (RpL36) presenting fixed differences between $L$. intermedia (blue) and $L$. whitmani (red). The circles are proportional to the haplotype frequencies and the black and grey lines connecting the haplotypes represent the number of mutational steps.

between the species seems intuitively reasonable, since the distribution of $L$. whitmani is wider than $L$. intermedia. L. whitmani is distributed throughout most of Brazil occurring also in neighboring countries. L. intermedia occurs in the Northeastern and part of the Southeastern regions of Brazil [23-25].

Although genealogies can be difficult to interpret in case of recombination, phylogenetic analyses do not have to assume any historical demographical model [26], and therefore it is useful to observe the gene trees and compare them with our summary-statistics analyses. Figures 3 and 4 illustrate the difference between the topologies of loci presenting either evidence for gene flow or high differentiation between species.

Three loci present fixed differences between $L$. intermedia and $L$. whitmani and no evidence of gene flow and can therefore be suggested as molecular markers to differentiate the two species: Rp49, RpS19a and RpL36. The latter also presents insertions/deletions which have not been considered in Table 2 . These three genes codify ribosomal protein subunits and it is intriguing that only such genes present fixed differences. As far as we know there is no reason one should expect these highly conserved ribosomal proteins to be less prone to introgression or to be under directional selection that would increase the likelihood of fixed differences between the two siblings. One interesting, but perhaps unlikely, possibility is that codon bias, as observed in Drosophila ribosomal protein genes [27], might somehow be responsible for the observed fixed differences.

The cac and per genes determine aspects of the lovesong patterns in Drosophila (reviewed in [28]), and therefore they are potentially good candidates for speciation loci in insects producing acoustic signals. So far, there is no behavioral study providing evidence that lovesongs play a role in the reproductive isolation between $L$. intermedia and L. whitmani as might be the case for the Lutzomyia longipalpis s.l. siblings $[29,30]$. Our data suggest the occurrence of introgression in cac and, although no evidence for gene flow in per was obtained in the present study, a previous analysis using this gene as a molecular marker also suggests introgression between L. intermedia and L. whitmani in at least one of the populations analyzed [9]. This evidence of gene flow in cac and per might indicate that these two loci do not have an important role, if any, in the reproductive isolation of $L$. intermedia and $L$. whitmani.

The RpL17A gene presented great differences between the genealogies and haploytpe networks obtained with the whole fragment and the NR blocks. While the latter show topologies with no grouping pattern for $L$. intermedia or $L$. whitmani and some identical haplotypes in both species (Figs. 3 and 4), the NJ tree and haplotype network from the whole fragment divide the species in two distinct groups (additional files 2 and 3 ). The same difference can be noticed for the $F_{s t}$ values, 0.4135 and 0.1749 for the whole fragment and NR block, respectively (Table 2). The IM simulations using the NR blocks indicated migration in the direction of $L$. whitmani at this gene. The observation that non-recombining blocks present low-divergence between species in opposition to the whole gene, suggests the occurrence of migration followed by recombination, where new haplotypes are created by recombining different alleles inside a population instead of by the occurrence of new mutations. It also reinforces the idea that different regions of a gene might have different evolutionary histories [3].

The zetacop gene has also presented interesting results. The fragment analyzed here revealed a large level of gene flow between the two species, with low (whole fragment) or non significant values (NR block) of $F_{s t}$ (Table 2) in addition to genealogies and networks that do not follow any 
grouping pattern (NR block in Figs. 3 and 4; whole fragment in additional files 2 and 3 ).

The epidemiological consequences of gene flow involving disease vectors are still not well known [31], however some studies do suggest important phenotypical changes in insect vectors due to introgression (see Background). The results obtained here are especially important because L. intermedia and L. whitmani are considered two main vectors of the cutaneous leishmaniasis, a disease for which the distribution has been expanding throughout Brazil, as silvatic areas have been constantly modified by urbanization [32-34]. The two species show some behavioral and ecological differences. L. intermedia is usually more common in the peridomestic area and more frequent in the summer months while L. whitmani is found mainly in the surrounding forest and is more abundant in the winter [33]. Interestingly it has been reported that only L. whitmani populations sympatric with $L$. intermedia are involved in cutaneous leishmaniasis transmission in the peridomestic environment [8]. The fact that we find evidence for introgression from $L$. intermedia towards $L$. whitmani is consistent with that although the loci we studied are probably not directly related to aspects of vectorial capacity. Further and more specific analyses focusing on the correlation between genetic, behavioral and habitat characteristics of these two sand fly species will be necessary to elucidate the possible consequences of gene flow for the disease epidemiology.

\section{Conclusion}

In conclusion, we have found evidence of introgression from $L$. intermedia towards L. whitmani in three out of ten analyzed loci. In addition, a different study using the per gene to analyze the geographical variation among populations of the two species has also indicated introgression in this locus. These findings suggest the occurrence of gene flow in roughly one third of the genome of these two vectors of cutaneous leishmaniasis in Southeastern Brazil raising the question whether this might be related to the observed changes in the epidemiological patterns of this disease.

\section{Methods \\ Choice of molecular markers}

Four loci selected for this analysis (Ca1D, cac, Rp49 and per) had been previously isolated from Lutzomyia species in our lab [35-38]. To select additional molecular markers to use in our study, a screen of cDNA sequences of $L$. longipalpis available in our lab was carried out. Genes with high similarity at the protein level compared to Anopheles gambiae and/or Drosophila melanogaster, and which present at least one intron in the region of aligned fragments, have been preferentially selected under the assumption that they have a well known function and potentially present an intron in Lutzomyia. Out of the 280 cDNA sequences initially checked, 26 were selected for primer design for PCR amplification. After amplification tests using genomic DNA of $L$. intermedia and $L$. whitmani, we ended up with 6 loci (RpL17A, RpL36, RpS19a, TfIIA-L, up, zetacop). Primers sequences of all ten loci used in our multilocus analysis are presented in Table 5.

\section{Sand fly samples and DNA methods}

Sand flies were collected in September 2004 from the locality of Afonso Claudio (Espírito Santo State, $20^{\circ} 04^{\prime} \mathrm{S}$ $41^{\circ} 07^{\prime} \mathrm{W}$ ), Southeast of Brazil, where L. intermedia and $L$. whitmani occur in sympatry. This locality is far from the known range of $L$. neivai, a third sibling closely related to the other two species that occurs mainly in the Southern and Central Western regions of Brazil and in neighboring countries $[8,24]$. L. intermedia and L. whitmani were identified according to Young and Duncan [23]. DNA was extracted from 96 individuals from each species according to Jowett [39] with slight modifications. A mix including $1 \mu$ of each individual DNA preparation was prepared for each species, and then used for PCR amplifications for all tested loci using Tth DNA polymerase (Biotools). PCR products were cloned in pMOS Blue Ended Vector (GE Healthcare) and sequenced at Fundação Oswaldo Cruz with an ABI 3730 DNA Sequencer, using "BigDye Terminators" (Applied Biosystems).

\section{Sequence analyses}

Sequences were edited using Wisconsin Package Version 9.1, Genetics Computer Group (GCG) and aligned using ClustalX [40]. Sites within indels or ambiguous alignment were removed before the analyses (see Table 5 ). Polymerase error rate was estimated as $1.56 \times 10^{-3}$ per nt. Based on this error rate, putative PCR induced singletons were randomly selected and corrected. Analyses of population polymorphisms and differentiation between populations were carried out using DNAsp4.1 [17] and ProSeq [41] softwares. The former program was also used for the recombination analysis. Table 5 shows the position of the non-recombining (NR) blocks used in this study as well as the sequences removed before the analyses carried out using these fragments. Neutrality tests were carried out using the HKA software [18] and DNAsp4.1.

Markov Chain Monte Carlo (MCMC) simulations of the isolation with migration model were carried out using the IM program [2]. IM estimates the marginal posterior probability for 6 demographic parameters from multilocus data, using an implementation of the MCMC (Monte Carlo Markov Chain) algorithm: time of divergence between the species $(t)$, effective population sizes for each species $\left(\theta_{1}\right.$ and $\left.\theta_{2}\right)$ and the ancestral population $\left(\theta_{\mathrm{A}}\right)$, and migration rates in both directions $\left(m_{1}\right.$ and $\left.m_{2}\right)$. The Infinite Sites model [42] has been chosen as the mutation 
Table 5: List of primers and edition of sequences

\begin{tabular}{|c|c|c|c|c|}
\hline locus & primers & Removed gap sites ${ }^{\mathrm{a}}$ & NR blocks ${ }^{b}$ & $\begin{array}{c}\text { Removed sequences from NR } \\
\text { blocksc }\end{array}$ \\
\hline CaID & $\begin{array}{c}\text { 5LWIca ID } \\
\text { 5'-CAGGATATAATGATGGATTG-3' } \\
\text { 3LWIca ID } \\
\text { 5'-CACGAACAAGTTGATAAT-3' }\end{array}$ & $159-160 ; 162-169 ; 183$ & $1-176$ & $\begin{array}{c}\text { IAFCa_AI 2; IAFCa_F09; } \\
\text { WAFCa_BI2; WAFCa_C09; } \\
\text { WAFCa_HII; WAFCa_HI2 }\end{array}$ \\
\hline Cac & $\begin{array}{c}\text { 5Llcac } \\
\text { 5'-GTGGCCGAACATAATGTTAG-3' } \\
\text { 3Llcac } \\
\text { 5'-CCACGAACAAGTTCAACATC-3' }\end{array}$ & $10 ; 19 ; 124-126 ; 129-132 ; 150 ; 170 ; 180 ; 182$ & $122-172$ & $\begin{array}{c}\text { IAFcac_H05; WAFcac_FOI; } \\
\text { WAFcac_H07 }\end{array}$ \\
\hline Per & $\begin{array}{c}\text { 5llper2 } \\
\text { 5'-AGCATCCTTTTGTAGCAAAC-3' } \\
\text { 3llper2 } \\
\text { 5'-TCAGATGAACTCTTGCTGTC-3' }\end{array}$ & I54-I57; 167-169; 204 & $125-210$ & IAFPERI6; WAFPER05 \\
\hline Rp49 & $\begin{array}{c}\text { 5RP49semidegl } \\
\text { 5'-TTCATYCGYCAYCAGWSBGA-3' } \\
\text { 3IIRP49exp2 } \\
\text { 5'-GGGCGATCTCAGCACAGTAT-3' }\end{array}$ & $29-36 ; 46-49 ; 55-56 ; 59 ; 65-69 ; 72 ; 74 ; 87$ & $\mathrm{I}-237$ & $\begin{array}{l}\text { IAFrp49_B08; IAFrp49_C08; } \\
\text { IAFrp49_E05; IAFrp49_E08; } \\
\text { IAFrp49_G08; IAFrp49_H06; } \\
\text { WAFrp49_DII }\end{array}$ \\
\hline RpLI 7A & $\begin{array}{c}\text { 5LLrpLI7A } \\
\text { 5'-TCAATTGCGCCGACAATAC-3' } \\
\text { 3LLrpLI7A } \\
\text { 5'-GCTGATCCTTTCATTTCGCC-3' }\end{array}$ & $\begin{array}{c}\text { 90-91; 10I-102; 109-II0; I3I; 135; 144; } \\
\text { I47-148; I53-154 }\end{array}$ & $1-114$ & $\begin{array}{l}\text { IAFrI7a_DI0; IAFrI7a_EIO; } \\
\text { IAFrI7a_FIO; IAFrI7a_GII }\end{array}$ \\
\hline RpL36 & $\begin{array}{c}\text { 5LWIrpL36 } \\
\text { 5'-GTTCCTCACGCTTCCTCTTG-3' } \\
\text { 3LWIrpL36 } \\
\text { 5'-AAAGTGAAAGGACTCCGCCC-3' }\end{array}$ & $\begin{array}{c}26-29 ; 46 ; 52 ; 65 ; 77 ; 215-217 ; 226 ; 238-253 ; \\
267 ; 270 ; 274 ; 289-294 ; 362\end{array}$ & $126-238$ & IAFr36_D07; IAFr36_HII \\
\hline RpSI9a & $\begin{array}{c}\text { 5LWrpSI9 } \\
\text { 5'-TGATCAACACAAGATTGTCCG-3' } \\
\text { 3LWrpSI9 } \\
\text { 5'-ACACCATTCCTCTTACGACC-3' }\end{array}$ & 174 & $77-193$ & $\begin{array}{c}\text { IAFI9_G0I; IAFI9_H02; } \\
\text { WAFI9_B08; WAFI9_F079 }\end{array}$ \\
\hline TflIA-L & $\begin{array}{c}\text { 5LLTfIIA-L } \\
\text { 5'-GATAATGATCCAGACGATGCC-3' } \\
\text { 3LLTfIIA-L } \\
\text { 5'-GAAAACATAGTCCTTCCCACC-3' }\end{array}$ & $\begin{array}{c}\text { I62; I73-I79; 20I-259; 277; 284-319; 34I; } \\
345-346 ; 369-370 ; 386-387\end{array}$ & $\mathrm{I}-254$ & - \\
\hline$U_{p}$ & $\begin{array}{l}\text { 5LLup } \\
\text { 5'-GCAACAAGTCCAAAGAGCAG-3' } \\
\text { 3LLup } \\
\text { 5'-TCATAGGAGCGGGTGTCAAC-3' }\end{array}$ & $\begin{array}{c}|89-19| ; 224 ; 35|-355 ; 360-36| ; 368-369 ; \\
396-397\end{array}$ & $\mathrm{I}-354$ & - \\
\hline zetacop & $\begin{array}{c}\text { 5LLzetacop } \\
\text { 5'-GGATGCAGATCCTTCATCCG-3' } \\
\text { 3LLzetacop } \\
\text { 5'-CGACCACTTCAGTTGTTCTC-3' }\end{array}$ & $\begin{array}{c}\text { II } 5 ; 131-134 ; 149-150 ; 168 ; 181 ; 195 ; 224 \\
234-235 ; 240-242\end{array}$ & $1-230$ & IAFztp_H07; WAFztp_C06 \\
\hline
\end{tabular}

a Sites with indels or ambiguous alignment removed before the analyses.

b Fragment positions of the non-recombining blocks used in the analyses.

c Recombining sequences removed before the analyses using the non-recombining blocks.

model since these are closely related species and all genes are nuclear. Upper limits were set to prior values for each of the demographic parameters based on preliminary runs. All values in the range were considered to present equal probabilities. Runs have been repeated at least three times using different seed numbers and a large number of updates ( 20 million) in order to guarantee convergence.

In addition to the simulations presented in the Results and Discussion section, tests using twice and half of the estimated number of singletons have been performed for IM analysis, in order to examine the effect of changes caused by differences in the number of corrected single- tons. The results indicate small quantitative and no qualitative differences in the parameter estimates. The migration estimates did not change for $m 2$ and show small differences for $m 1$, around 0.018 in either direction.

\section{Authors' contributions}

CJM, ASA, and RVDMA generated the data. CJM analyzed all the data and drafted the manuscript. ASA and GEMF collected sand fly samples. GB helped to write the manuscript and supervised CJM during her stay in Italy. AAP is the principal investigator, participated in its design and coordination, and helped to write the manuscript. All authors read and approved the final manuscript. 


\section{Additional material}

\section{Additional file 1}

Supplemental Table - Tests of neutrality. The table presents the results of neutrality tests $\mathrm{Fu}$ and Li's $D^{*}$ and $F^{*}, \mathrm{Fu}$ 's $F_{S}$ and Ramos-Onsins and Rozas' $R_{2}$.

Click here for file

[http://www.biomedcentral.com/content/supplementary/14712148-8-141-S1.doc]

\section{Additional file 2}

Genealogies of RpL17A and zetacop whole fragments. The figure shows trees of RpL17A and zetacop sequences (whole fragments) of L. intermedia (blue circles) and $\mathrm{L}$. whitmani (red squares). The trees were estimated using the neighbor-joining method, Kimura-2-parameters distance and 1000 bootstrap replicates, and rooted using the middle-point between the two most distant sequences.

Click here for file

[http://www.biomedcentral.com/content/supplementary/14712148-8-141-S2.pdf]

\section{Additional file 3}

Haplotype networks of RpL17A and zetacop whole fragments. The figure shows minimum spanning trees of RpL17A and zetacop sequences (whole fragments) of L. intermedia (blue) and L. whitmani (red). The circles are proportional to the haplotype frequencies and the black and grey lines connecting the haplotypes represent the number of mutational steps. Click here for file

[http://www.biomedcentral.com/content/supplementary/14712148-8-141-S3.pdf]

\section{Acknowledgements}

We thank Denise Dias for providing some of the unpublished $L$. longipalpis cDNA sequences used in the initial screen and Claudiney Biral dos Santos for assistance with sand fly collections. This work was funded by the Howard Hughes Medical Institute with additional support from FiocruzPAPES IV, Capes and CNPq.

\section{References}

I. Coyne JA, Orr HA: Speciation Sunderland: Sinauer Associates; 2004.

2. Hey J, Nielsen R: Multilocus methods for estimating population sizes, migration rates and divergence time, with applications to the divergence of Drosophila pseudoobscura and D. persimilis. Genetics 2004, I 67:747-760.

3. Machado CA, Kliman RM, Markert JA, Hey J: Inferring the history of speciation from multilocus DNA sequence data: the case of Drosophila pseudoobscura and close relatives. Mol Biol Evol 2002, 19:472-488.

4. Fonseca DM, Keyghobadi N, Malcolm CA, Mehmet C, Schaffner F, Mogi M, Fleischer RC, Wilkerson RC: Emerging vectors in the Culex pipiens complex. Science 2004, 303:I535-I538.

5. Besansky NJ, Krzywinski J, Lehmann T, Simard F, Kern M, Mukabayire O, Fontenille D, Toure Y, Sagnon N: Semipermeable species boundaries between Anopheles gambiae and Anopheles arabiensis: evidence from multilocus DNA sequence variation. Proc Natl Acad Sci USA 2003, I00:10818-10823.

6. Esseghir S, Ready P, Ben-Ismail R: Speciation of Phlebotomus sandflies of the subgenus Larroussius coincided with the late Miocene-Pliocene aridification of the Mediterranean subregion. Biol J Linn Soc 2000, 70:189-219.

7. Testa JM, Montoya-Lerma J, Cadena H, Oviedo M, Ready PD: Molecular identification of vectors of Leishmania in Colombia: mitochondrial introgression in the Lutzomyia townsendi series. Acta Trop 2002, 84:205-218.
8. Marcondes CB, Day JC, Ready PD: Introgression between Lutzomyia intermedia and both Lu. neivai and Lu. whitmani, and their roles as vectors of Leishmania braziliensis. Trans $R$ Soc Trop Med Hyg 1997, 91:725-726.

9. Mazzoni CJ, Souza NA, Andrade-Coelho C, Kyriacou CP, Peixoto $A A$ : Molecular polymorphism, differentiation and introgression in the period gene between Lutzomyia intermedia and Lutzomyia whitmani. BMC Evol Biol 2006, 6:85.

10. Peixoto AA: Evolutionary behavioral genetics in Drosophila. Adv Genet 2002, 47:1 17-150.

II. Flybase [http://www.flybase.org]

12. Hudson RR, Kaplan NL: Statistical properties of the number of recombination events in the history of a sample of DNA sequences. Genetics 1985, I I I: 147-164.

13. Tajima F: Statistical method for testing the neutral mutation hypothesis by DNA polymorphism. Genetics 1989, I 23:585-595.

14. Fu Y-X, Li W-H: Statistical tests of neutrality of mutations. Genetics 1993, I 33:693-709.

15. Ramos-Onsins SE, Rozas J: Statistical properties of new neutrality tests against population growth. Mol Biol Evol 2002, 19:2092-2100.

16. Fu Y-X: Statistical tests of neutrality of mutations against population growth, hitchhiking and background selection. Genetics 1997, I 47:915-925.

17. Rozas J, Sánchez-DelBarrio JC, Messeguer X, Rozas R: DnaSP, DNA polymorphism analyses by the coalescent and other methods. Bioinformatics 2003, 19:2496-2497.

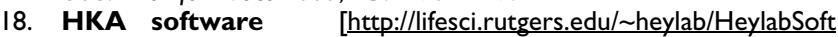
ware.htm]

19. Hudson RR, Kreitman M, Aguade M: A test of neutral molecular evolution based on nucleotide data. Genetics 1987 I 1 6:153-159.

20. Li WH: Molecular Evolution Sunderland: Sinauer Associates; 1997.

21. Kumar S, Tamura K, Nei M: MEGA3: Integrated Software for Molecular Evolutionary Genetics Analysis and Sequence Alignment. Briefings in Bioinformatics 2004, 5:150-163.

22. Nielsen R, Wakeley J: Distinguishing migration from isolation: a Markov chain Monte Carlo approach. Genetics 200I, I 58:885-896.

23. Young $D$, Duncan MA: Guide to the identification and geographic distribution of Lutzomyia sandflies in Mexico, the West Indies, Central and South America (Diptera: Psychodidae). Mem Am Entomol Inst 1994, 54: I-88I.

24. Andrade-Filho JD, Galati EA, Falcao AL: Nyssomyia intermedia (Lutz \& Neiva, 19/2) and Nyssomyia neivai (Pinto, 1926) (Diptera: Psychodidae: Phlebotominae) geographical distribution and epidemiological importance. Mem Inst Oswaldo Cruz 2007, I 02:48|-487.

25. da Costa SM, Cechinel M, Bandeira V, Zannuncio JC, Lainson R, Rangel EF: Lutzomyia (Nyssomyia) whitmani s.l. (Antunes \& Coutinho, 1939) (Diptera: Psychodidae: Phlebotominae): geographical distribution and the epidemiology of American cutaneous leishmaniasis in Brazil - Mini-review. Mem Inst Oswaldo Cruz 2007, I02:149-153.

26. Hey J, Machado CA: The study of structured populations - new hope for a difficult and divided science. Nat Rev Genet 2003, 4:535-543.

27. Heger A, Ponting CP: Variable strength of translational selection among 12 Drosophila species. Genetics 2007, I 77:1337-1348.

28. Gleason JM: Mutations and natural genetic variation in the courtship song of Drosophila. Behav Genet 2005, 35:265-277.

29. de Souza NA, Ward RD, Hamilton JG, Kyriacou CP, Peixoto AA: Copulation songs in three siblings of Lutzomyia longipalpis (Diptera: Psychodidae). Trans R Soc Trop Med Hyg 2002, 96:102-103.

30. Souza NA, Vigoder FM, Araki AS, Ward RD, Kyriacou CP, Peixoto AA: Analysis of the copulatory courtship songs of Lutzomyia longipalpis in six populations from Brazil. J Med Entomol 2004, 41:906-913.

31. Donnelly MJ, Pinto J, Girod R, Besansky NJ, Lehmann T: Revisiting the role of introgression vs shared ancestral polymorphisms as key processes shaping genetic diversity in the recently separated sibling species of the Anopheles gambiae complex. Heredity 2004, 92:6I-68. 
32. Campbell-Lendrum D, Dujardin JP, Martinez E, Feliciangeli MD, Perez JE, Silans LN, Desjeux P: Domestic and peridomestic transmission of American cutaneous leishmaniasis: changing epidemiological patterns present new control opportunities. Mem Inst Oswaldo Cruz 200I, 96:159-162.

33. Souza NA, Andrade-Coelho CA, Vilela ML, Peixoto AA, Rangel EF: Seasonality of Lutzomyia intermedia and Lutzomyia whitmani (Diptera: Psychodidae: Phlebotominae), occurring sympatrically in area of cutaneous leishmaniasis in the State of Rio de Janeiro, Brazil. Mem Inst Oswaldo Cruz 2002, 97:759-765.

34. Dias ES, França-Silva JC, Silva JC, Monteiro ÉM, Paula KM, Gonçalves CM, Barata RA: Flebotomíneos (Diptera: Psychodidae) de um foco de leishmaniose tegumentar no Estado de Minas Gerais. Rev Soc Bras Med Trop 2007, 40:49-52.

35. Peixoto AA, Gomes CA, de Amoretty PR, Lins RM, Meireles-Filho AC, Souza NA, Kyriacou CP: New molecular markers for phlebotomine sand flies. Int J Parasitol 200I, 3 I:635-639.

36. Lins RM, Oliveira SG, Souza NA, de Queiroz RG, Justiniano SC, Ward RD, Kyriacou CP, Peixoto AA: Molecular evolution of the cacophony IVS6 region in sandflies. Insect Mol Biol 2002, II:I17-122.

37. Mazzoni CJ, Gomes CA, Souza NA, de Queiroz RG, Justiniano SC, Ward RD, Kyriacou CP, Peixoto AA: Molecular evolution of the period gene in sandflies. J Mol Evol 2002, 55:553-562.

38. Meireles-Filho AC, SRGB, Gesto JS, Machado RC, Britto C, Souza NA, Peixoto AA: The biological clock of an hematophagous insect: locomotor activity rhythms, circadian expression and downregulation after a blood meal. FEBS Letters 2006, 580:2-8.

39. Jowett T: Preparation of nucleic acids. In Drosophila: A practical approach Edited by: Roberts DB. Oxford: IRL press; 1998:347-37I.

40. Thompson JD, Gibson T], Plewniak F, Jeanmougin F, Higgins DG: The ClustalX windows interface: flexible strategies for multiple sequence alignment aided by quality analysis tools. Nucleic Acids Res 1997, 24:4876-4882.

41. Filatov DA: A software for preparation and evolutionary analysis of DNA sequence data sets. Mol Ecol Notes 2002, 2:62। -624.

42. Kimura $M$ : The number of heterozygous nucleotide sites maintained in a finite population due to steady flux of mutations. Genetics 1969, 61:893-903.

Publish with Bio Med Central and every scientist can read your work free of charge

"BioMed Central will be the most significant development for disseminating the results of biomedical research in our lifetime. "

Sir Paul Nurse, Cancer Research UK

Your research papers will be:

- available free of charge to the entire biomedical community

- peer reviewed and published immediately upon acceptance

- cited in PubMed and archived on PubMed Central

- yours - you keep the copyright
BioMedcentral 